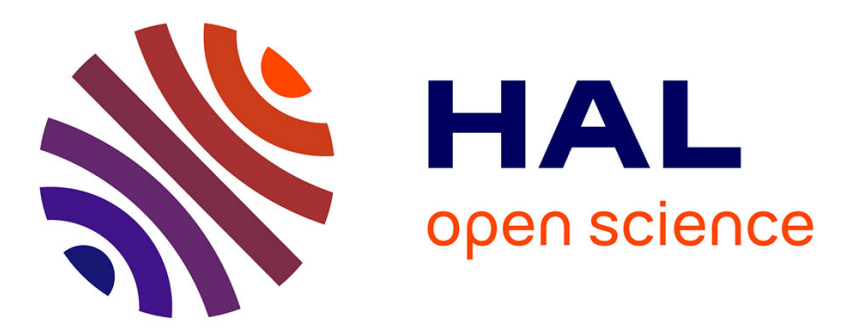

\title{
New implementions of predictive alternate analog/RF test with augmented model redundancy
}

Haithem Ayari, Florence Azaïs, Serge Bernard, Mariane Comte, Vincent Kerzérho, Michel Renovell

\section{- To cite this version:}

Haithem Ayari, Florence Azaïs, Serge Bernard, Mariane Comte, Vincent Kerzérho, et al.. New implementions of predictive alternate analog/RF test with augmented model redundancy. DATE 2014 - 17th Design, Automation and Test in Europe Conference and Exhibition, Mar 2014, Dresden, Germany. 10.7873/DATE2014.144 . lirmm-00994714

\section{HAL Id: lirmm-00994714 \\ https://hal-lirmm.ccsd.cnrs.fr/lirmm-00994714}

Submitted on 22 May 2014

HAL is a multi-disciplinary open access archive for the deposit and dissemination of scientific research documents, whether they are published or not. The documents may come from teaching and research institutions in France or abroad, or from public or private research centers.
L'archive ouverte pluridisciplinaire HAL, est destinée au dépôt et à la diffusion de documents scientifiques de niveau recherche, publiés ou non, émanant des établissements d'enseignement et de recherche français ou étrangers, des laboratoires publics ou privés. 


\title{
New implementions of predictive alternate analog/RF test with augmented model redundancy
}

\author{
H. AYARI, F. AZAIS, S. BERNARD, M. COMTE, V. KERZERHO and M. RENOVELL \\ LIRMM, CNRS/Univ. Montpellier 2 \\ Montpellier, France
}

\begin{abstract}
This paper discusses new implementations of the predictive alternate test strategy that exploit model redundancy in order to improve test confidence. The key idea is to build during the training phase, not only one regression model for each specification as in the classical implementation, but several regression models. This redundancy is then used during the testing phase to identify suspect predictions and remove the corresponding devices from the alternate test flow. In this paper, we explore various options for implementing model redundancy, based on the use of different indirect measurement combinations and/or different partitions of the training set. The proposed implementations are evaluated on a real case study for which we have production test data from 10,000 devices.
\end{abstract}

Keywords - Test; analog/RF integrated circuits; alternate test, specification prediction, test confidence

\section{INTRODUCTION}

The alternate test strategy is a promising solution to reduce the testing costs of analog/RF circuits. The basic idea of this strategy is to replace standard specification tests by a set of few alternate measurements obtained with low-cost test equipment, and to infer the results of specification testing using only these low-cost indirect measurements. This strategy therefore offers substantial test cost reduction by relaxing constraints on both the number and the complexity of required test configurations.

Alternate testing has been widely studied in the literature for many years [1-10]. Many aspects have been researched, such as the choice of the learning algorithm, the definition and optimization of appropriate test stimuli, the processing of complex signatures, the use of embedded sensors to gather pertinent information, the exploitation multi-Vdd test conditions and procedures for the selection of appropriate indirect measurements. Despite the number of good results reported in all these works that illustrate the efficiency of the alternate test strategy, its industrial deployment is still limited, mainly because of a confidence problem. To cope with this issue, an interesting approach is to use a two-tier test scheme in which devices for which the alternate test decision may be prone to error are identified and directed towards a different tier where further testing may apply [7]. Different solutions have been explored for the identification of suspect devices based either on kernel density estimation [8] or on the use of redundancy $[9,10]$. In this paper, we extend our previous work based on redundancy, and we explore various options for implementing model redundancy with the objective to strengthen the confidence in alternate test predictions.

\section{Alternate TeSt PRINCIPLE}

The underlying idea of alternate testing is that process variations that affect the conventional performance parameters of the device also affect non-conventional low-cost indirect parameters. If the correlation between the indirect parameter space and the performance parameter space can be established, then specifications may be verified using only the low-cost indirect signatures. Unfortunately the relation between these two sets of parameters is complex and cannot be simply identified with an analytic function. The solution commonly implemented uses machine-learning algorithms.

The alternate test principle is actually split into two sequential steps, namely training and production testing phases. The idea is to learn during the training phase the unknown dependency between the low-cost indirect parameters and the conventional test ones. For this, both the specification tests and the low-cost measurements are performed on a training set of device instances. The mapping derived from the training phase is then used during the production testing phase in order to perform device specification prediction or classification using only the low-cost indirect measurements. The classical implementation of predictive alternate testing is illustrated in Fig. 1.

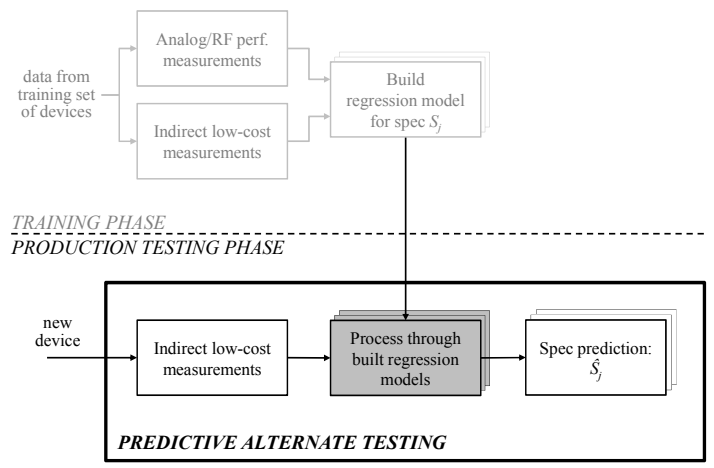

Figure 1. Predictive alternate test strategy

\section{PREVIOUS WORK AND LIMITATIONS}

The alternate test strategy offers interesting perspectives for a low-cost test solution of analog/RF circuits. However this strategy is still not widely used in industry today, mainly because of a problem of confidence. Indeed although experiments reported in the literature show that good prediction accuracy can be achieved on various devices, in 
particular in terms of average prediction error, there are two main points that limit the credit one can give to this good accuracy. First, low average prediction error does not guarantee low maximal prediction error, which is of crucial importance regarding the classification step where the predicted values are compared to the specification limits promised in the data sheet. Second, evaluation is usually performed on a small set of validation devices, typically ranging from few hundreds to one thousand instances, while the technique aims at predicting values for a large set of fabricated devices, typically one or several millions.

In our previous work [9], experiments performed on a Power Amplifier (PA) fabricated by NXP Semiconductors for which we have production test data from 10,000 devices have revealed that the large majority of devices can indeed be accurately predicted using the alternate test strategy, but rather large prediction errors are observed for some circuits. Although the number of devices affected by such large prediction error is very small, i.e. much less than $1 \%$, this is a serious obstacle for the deployment of the strategy in an industrial context. To cope with this issue, we have proposed a two-tier test scheme that includes an additional step during the testing phase in order to evaluate prediction confidence; if confidence is good, the device is evaluated using the low-cost alternate test tier otherwise it is directed to another tier for further testing. As illustrated in Fig. 2, the proposed scheme exploits model redundancy in order to order to distinguish reliable predictions from suspect predictions. More precisely, 3 regression models that involve different combinations of indirect measurements are built during the training phase for each specification. Then during the testing phase, prediction confidence is established by checking the consistency between the values predicted by the 3 different models, for each specification. For this, the difference between the predicted values is computed for each pair of models and checked against a threshold value $\varepsilon_{\text {thi }}$. If one (or more) of these differences is superior to $\varepsilon_{\mathrm{thi}}$, the prediction is considered suspect and the device is directed to the second tier where further testing may be applied. On the contrary, if all these differences are inferior to the threshold, the prediction is considered reliable and device specification is computed as the mean of the values predicted by the different models.

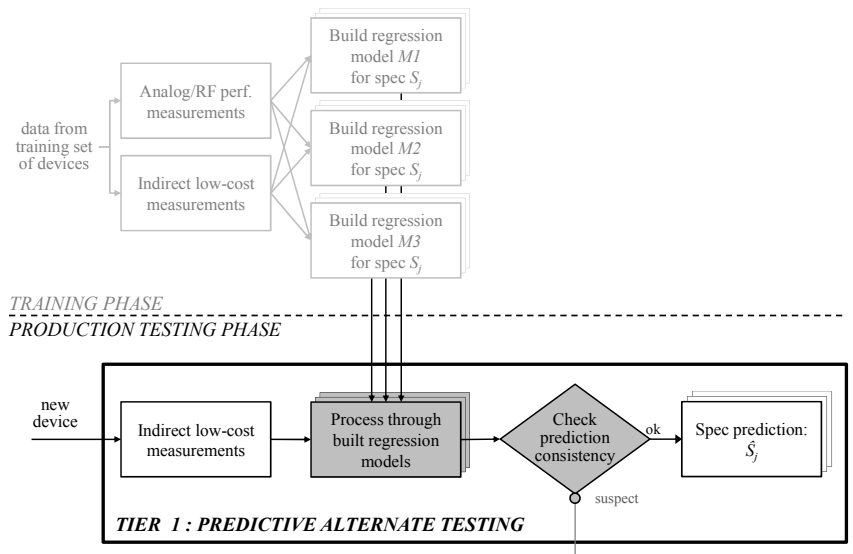

TIER 2 : FURTHER TESTING

Figure 2. Two-tier alternate test scheme using model redundancy
Validation results obtained on the power amplifier case study have shown that, compared to a classical alternate test implementation, this scheme permits to achieve better performances in terms of both average and maximal prediction errors for devices evaluated by the alternate test tier while only a small fraction of the devices are directed to the second tier therefore incurring low test cost overhead.

The efficiency of the proposed scheme relies on the assumption that it is very unlikely that regression models built using different combinations of indirect measurements will erroneously predict the device performance with the same error. To verify this assumption, we have conducted a number of experiments on the power amplifier case study. Available production test data include 37 low-cost Indirect Measurements (IMs) based on standard DC tests and two RF performance measurements, namely the $1 \mathrm{~dB}$ compression point (CP1) and 3rd order intercept point (IP3). These data are separated in two distinct sets of 5,000 devices, one used for training and the other for validation. Based on previous work showing that the RF performance parameters can be predicted with low average prediction error using models based only on few IMs [6], we have built regression models using all possible combinations of 3 IMs (7,770 models for each specification) and we have retained models with a relatively good accuracy (more than 5,000 models with an average prediction error below $2 \%$ for each specification). We have then evaluated the performances achieved by the proposed two-tier test scheme regarding CP1 specification when using 3 models randomly chosen among the retained models, repeating this experiment 100 times. As expected results show that, compared to the classical implementation with a single model, prediction errors are significantly reduced for all devices evaluated by the alternate test tier. However we denote one case where the procedure fails to identify a relatively large prediction error on one device of the validation set. For this case, the 3 regression models actually yield to an erroneous performance prediction with a similar error (difference between each pair of predicted values is less than $1 \mathrm{dBm}$ ) for one particular device. The prediction is therefore not detected as a suspect one and the device is evaluated using the alternate test tier. So this is one limitation of the proposed approach.

\section{Proposed IMPLEMENTATIONS WITH AUgMENTED MODEL REDUNDANCY}

Despite the use of 3 regression models based on different IM combinations, it may happen the 3 models erroneously predict a device performance with a similar error. In this case the procedure that checks prediction consistency is ineffective and the device is not directed towards the appropriate test tier. Our objective is to reinforce redundancy in order to avoid such situation. More precisely, the idea is to increase the number of redundant models used the procedure that checks prediction consistency in order to diminish the probability that all models give the same erroneous prediction for one device.

A first option to increase redundancy is to continue to exploit the different indirect measurements and to build, for each specification, not only 3 but a higher number of regression models based different IM combinations. However it is clear that selecting a higher number of different IM 
combinations will inevitably imply a higher number of indirect measurements that have to be performed, which has a direct impact on the testing costs. This option is therefore not favored. Instead, our idea is to exploit another attribute of the data available for the construction of regression models related to training devices. Indeed, data used for the construction of a regression model clearly involves two aspects, i.e. indirect measurements and training devices. Any change in the set of indirect measurements or in the set of training devices results in a different model. Up to now, redundant models have been built by changing the set of considered indirect measurements. The idea is maintain this feature but also to exploit the other aspect, i.e. to build redundant models by changing the set of training devices. In particular, the idea is to split the training set in a number of partitions and to build regression models for the different partitions.

Our proposal for the generation of redundant regression models, for each specification $S_{j}$, is summarized in Fig. 3. On one hand 3 combinations of indirect measurements are selected from the set of available indirect measurements, and on the other hand the training set is split is 3 distinct partitions. For each selected IM combination $C_{i}$, a regression model is then built considering the different partitions of the training set. A total number of 9 redundant models are therefore built, which exploit both different IM combinations and different partitions of the training set. These 9 models will be used during the testing phase in order to establish prediction confidence.

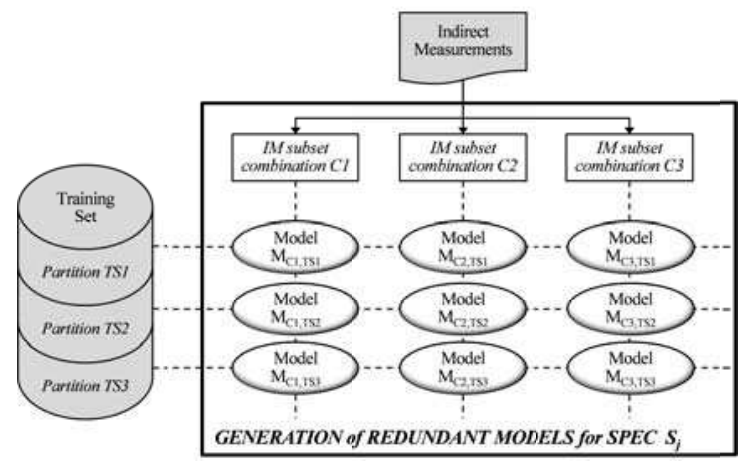

Figure 3. Generation of redundant regression models

We have actually explored different implementations of the procedure that Checks Prediction Consistency (CPC). In the first version, consistency between the values predicted by the 9 models is checked in a single step, i.e. the difference between the predicted values is computed for each pair of models and prediction is considered reliable only if all these 36 differences are inferior to a given threshold $\varepsilon_{\text {thi }}$. In the case, the final predicted value is computed as the mean of the 9 predicted values. In the two other versions, prediction consistency is verified is two steps. In the first step, consistency between predicted values is checked considering models 3 by 3 according to the used training set partition in one version or the used IM combination in the other version; 3 intermediate predicted values can then computed. In the second step, consistency between these 3 intermediate values is checked and the final predicted value is computed as the mean of these 3 values.

\section{EXPERIMENTAL RESULTS}

A number of experiments have been performed on the PA case study to validate the proposed strategy. First, we have evaluated the proposed implementations considering the particular example discussed in section III. Results are summarized in Table I that reports both average and maximal prediction errors observed for the different implementations. Note that implementations with augmented model redundancy involve the partitioning of the training set in 3 disjoint subsets. Although the training set comprises a rather large number of devices, results are quite sensitive to the repartition of training devices in the different subsets. Consequently, we create several random splits of the training devices in 3 partitions and we report minimum, mean and maximum values observed over all runs (100 runs in this experiment). Finally for the sake of comparison, we also report results obtained when using a reduced-cost implementation that exploits model redundancy based only on different training set partitions (a single IM combination is considered and only 3 redundant models are built using the 3 different partitions).

From the results of table I, it can be observed that the 3 versions of the new implementation of augmented model redundancy have equivalent performances. The efficiency of these new implementations is clearly demonstrated, since they permit to reduce average and maximal prediction errors compared to the initial implementation. The improvement is particularly significant regarding the maximal prediction error that reduces from $4.08 \mathrm{dBm}$ with the initial implementation down to less than $1 \mathrm{dBm}$. Also it can be observed that the costreduced implementation that exploits model redundancy based only on different training set partitions offers degraded performances compared to other implementations.

TABLE I. AVERAGE AND MAXIMAL PREDICTION ERRORS FOR THE PARTICULAR CP1 PREDICTION EXAMPLE OF SECTION III

\begin{tabular}{|c|c|c|c|c|c|c|}
\hline & & \multirow{2}{*}{$\begin{array}{l}\text { Initial } \\
\text { Implem. }\end{array}$} & \multicolumn{3}{|c|}{ New Implementations } & \multirow{2}{*}{$\begin{array}{l}\text { Cost- } \\
\text { reduced } \\
\text { Implem. }\end{array}$} \\
\hline & & & Version A & Version B & Version C & \\
\hline 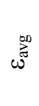 & $\begin{array}{c}\min \\
\text { mean } \\
\max \end{array}$ & $1.28 \%$ & $\begin{array}{l}1.17 \% \\
1.18 \% \\
1.19 \%\end{array}$ & $\begin{array}{l}1.17 \% \\
1.18 \% \\
1.19 \%\end{array}$ & $\begin{array}{l}1.17 \% \\
1.18 \% \\
1.20 \%\end{array}$ & $\begin{array}{l}1.65 \% \\
1.71 \% \\
1.76 \%\end{array}$ \\
\hline 弟 & $\begin{array}{c}\min \\
\text { mean } \\
\max \end{array}$ & $4.08 \mathrm{dBm}$ & $\begin{array}{l}0.77 \mathrm{dBm} \\
0.81 \mathrm{dBm} \\
0.96 \mathrm{dBm}\end{array}$ & $\begin{array}{l}0.77 \mathrm{dBm} \\
0.81 \mathrm{dBm} \\
0.96 \mathrm{dBm}\end{array}$ & $\begin{array}{l}0.78 \mathrm{dBm} \\
0.82 \mathrm{dBm} \\
0.96 \mathrm{dBm}\end{array}$ & $\begin{array}{l}2.03 \mathrm{dBm} \\
3.35 \mathrm{dBm} \\
5.09 \mathrm{dBm}\end{array}$ \\
\hline
\end{tabular}

Results on the previous CP1 prediction example are obtained considering 3 particular IM combinations. To further corroborate these results, we have conducted a large campaign of experiments varying the IM combinations used to build the redundant models. More precisely for each specification, we perform 50 random selections of 3 different IM combinations among all the combinations corresponding to models with a satisfying accuracy. For each 3 selected IM combinations, redundant models are then built considering 100 random splits of the training devices in 3 partitions. Implementations with augmented model redundancy are therefore evaluated over 5,000 different cases of generated redundant models. Results are summarized in Tables II and III for CP1 and IP3 predictions respectively. 
TABLE II. CP1 PREDICTION RESULTS COMPARISON OF THE DIFFERENT IMPLEMENTATIONS

\begin{tabular}{|c|c|c|c|c|c|c|}
\hline & & \multirow{2}{*}{$\begin{array}{l}\text { Initial } \\
\text { Implem. }\end{array}$} & \multicolumn{3}{|c|}{ New Implementations } & \multirow{2}{*}{$\begin{array}{l}\text { Cost- } \\
\text { reduced } \\
\text { Implem. }\end{array}$} \\
\hline & & & Version A & Version B & Version $\mathrm{C}$ & \\
\hline \multirow{4}{*}{$\omega^{\infty}$} & $\min$ & $0.69 \%$ & $0.66 \%$ & $0.66 \%$ & $0.66 \%$ & $0.64 \%$ \\
\hline & mean & $0.97 \%$ & $0.96 \%$ & $0.96 \%$ & $0.97 \%$ & $1.11 \%$ \\
\hline & $\max$ & $1.28 \%$ & $1.25 \%$ & $1.25 \%$ & $1.26 \%$ & $5.03 \%$ \\
\hline & $\sigma$ & $0.16 \%$ & $0.15 \%$ & $0.15 \%$ & $0.15 \%$ & $0.31 \%$ \\
\hline \multirow{4}{*}{ 弟 } & $\min$ & $0.70 \mathrm{dBm}$ & $0.52 \mathrm{dBm}$ & $0.53 \mathrm{dBm}$ & $0.53 \mathrm{dBm}$ & $0.66 \mathrm{dBm}$ \\
\hline & mean & $1.06 \mathrm{dBm}$ & $0.91 \mathrm{dBm}$ & $0.93 \mathrm{dBm}$ & $0.95 \mathrm{dBm}$ & $1.57 \mathrm{dBm}$ \\
\hline & $\max$ & $4.08 \mathrm{dBm}$ & $2.16 \mathrm{dBm}$ & $2.17 \mathrm{dBm}$ & $2.16 \mathrm{dBm}$ & $38.49 \mathrm{dBm}$ \\
\hline & $\sigma$ & $0.42 \mathrm{dBm}$ & $0.28 \mathrm{dBm}$ & $0.29 \mathrm{dBm}$ & $0.29 \mathrm{dBm}$ & $0.78 \mathrm{dBm}$ \\
\hline
\end{tabular}

TABLE III. IP3 PREDICTION RESULTS COMPARISON OF THE DIFFERENT IMPLEMENTATIONS

\begin{tabular}{|c|c|c|c|c|c|c|}
\hline & & \multirow{2}{*}{$\begin{array}{l}\text { Initial } \\
\text { Implem. }\end{array}$} & \multicolumn{3}{|c|}{ New Implementations } & \multirow{2}{*}{$\begin{array}{c}\text { Cost- } \\
\text { reduced } \\
\text { Implem. }\end{array}$} \\
\hline & & & Version A & Version B & Version $\mathrm{C}$ & \\
\hline \multirow{4}{*}{$\stackrel{\infty}{\omega^{\infty}}$} & $\min$ & $0.87 \%$ & $0.85 \%$ & $0.85 \%$ & $0.85 \%$ & $0.91 \%$ \\
\hline & mean & $1.06 \%$ & $1.06 \%$ & $1.06 \%$ & $1.06 \%$ & $1.17 \%$ \\
\hline & $\max$ & $1.31 \%$ & $1.41 \%$ & $1.43 \%$ & $1.44 \%$ & $1.58 \%$ \\
\hline & $\sigma$ & $0.07 \%$ & $0.07 \%$ & $0.07 \%$ & $0.07 \%$ & $0.09 \%$ \\
\hline \multirow{4}{*}{ 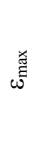 } & $\min$ & $1.01 \mathrm{dBm}$ & $0.97 \mathrm{dBm}$ & $0.97 \mathrm{dBm}$ & $0.97 \mathrm{dBm}$ & $1.17 \mathrm{dBm}$ \\
\hline & mean & $1.77 \mathrm{dBm}$ & $1.51 \mathrm{dBm}$ & $1.55 \mathrm{dBm}$ & $1.59 \mathrm{dBm}$ & $2.93 \mathrm{dBm}$ \\
\hline & $\max$ & $7.35 \mathrm{dBm}$ & $4.47 \mathrm{dBm}$ & $6.28 \mathrm{dBm}$ & $6.50 \mathrm{dBm}$ & $14.66 \mathrm{dBm}$ \\
\hline & $\sigma$ & $0.96 \mathrm{dBm}$ & $0.50 \mathrm{dBm}$ & $0.53 \mathrm{dBm}$ & $0.56 \mathrm{dBm}$ & $1.74 \mathrm{dBm}$ \\
\hline
\end{tabular}

These results confirm the superiority of the new implementations with augmented model redundancy. Indeed although impact on the average prediction error is not significant, there is a substantial improvement regarding the maximal prediction error, with a reduction not only of the mean and maximum values observed over the different cases of generated redundant models, but also of the standard deviation. In particular, this standard deviation reduces from $0.42 \mathrm{dBm}$ with the initial implementation down to $0.28 \mathrm{dBm}$ for $\mathrm{CP} 1$ specification, and from $0.96 \mathrm{dBm}$ down to $0.5 \mathrm{dBm}$ for IP3 specification. This constitutes an important improvement of the robustness of the technique. Regarding comparison between the performances offered by the 3 versions of the new implementation, they are equivalent for CP1 specification, but version A leads to slightly better results for IP3 specification; this implementation may therefore be preferred. Regarding the cost-reduced implementation that exploit model redundancy based only on different training set partitions, this solution should not be retained as it presents degraded performances compared to other implementations.

Finally we have compared the different implementations regarding the percentage of circuits for which suspect predictions are identified. Results are summarized in Fig. 4. As expected, implementations with augmented model redundancy lead to a higher number of suspect predictions compared to implementations with model redundancy based only on either different IM combinations or different training set partitions, both for CP1 and IP3 specifications. However the percentage remains extremely low, less than $1.5 \%$, which means that the new implementations permits to improve prediction results while maintaining a low test cost overhead.

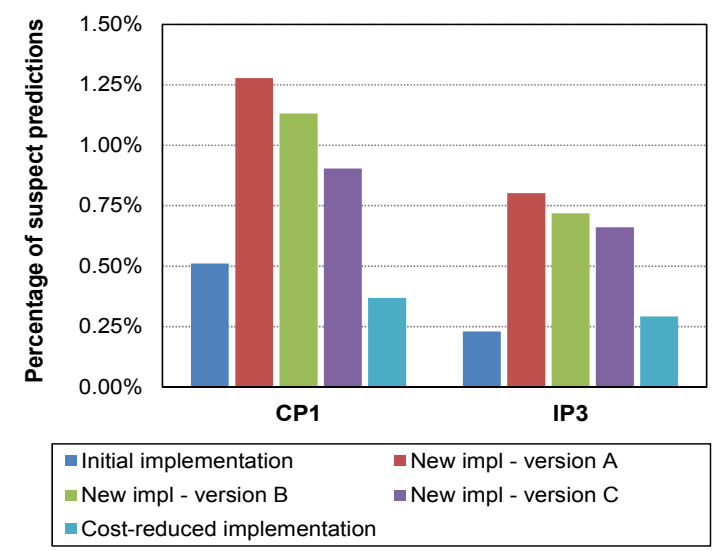

Figure 4. Comparison of different implementations in tems of percentage of suspect predictions

\section{CONCLUSION}

In this paper we have proposed new implementations of the predictive alternate test strategy that exploit model redundancy. These new implementations are based on redundant models that involve not only different combinations of indirect measurements as initially proposed, but also different partitions of the training set. Combining these two aspects permits the generation of a higher number of redundant models while maintaining the same cost, i.e. the same number of indirect measurements to be performed. The different implementations have been evaluated on a real case study for which we have production test data from 10,000 devices. Results have shown that implementations with reinforced model redundancy permit to reduce prediction errors of circuits evaluated by the alternate test tier, while maintaining a very small number of devices directed to a second tier for further testing.

\section{REFERENCES}

[1] Variyam, P.N.; Chatterjee, A.; , "Enhancing test effectiveness for analog circuits using synthesized measurements," IEEE VLSI Test Symposium, pp.132-137, 26-30 Apr 1998

[2] Variyam, P.N.; Cherubal, S.; Chatterjee, A.; , "Prediction of analog performance parameters using fast transient testing," IEEE Trans on CAD of Int. Circuits \& Systems, vol.21, no.3, pp.349-361, Mar 2002

[3] Ellouz, S et al.; "Combining Internal Probing with Artificial Neural Networks for Optimal RFIC Testing," IEEE Int'l Test Conference (ITC), pp.1-9, 2006

[4] Abdallah, L.; et al., "Sensors for built-in alternate RF test," IEEE European Test Symp (ETS), pp.49-54, 2010

[5] Barragan, M.J et al. , "Improving the Accuracy of RF Alternate Test Using Multi-VDD Conditions: Application to Envelope-Based Test of LNAs," IEEE Asian Test Symposium (ATS), pp.359-364, 2011

[6] Ayari, H.; et al.., "Smart selection of indirect parameters for DC-based alternate RF IC testing," IEEE VLSI Test Symp (VTS), pp.19-24, 2012

[7] Stratigopoulos, H.-G.; Makris, Y.; , "Error Moderation in Low-Cost Machine-Learning-Based Analog/RF Testing," IEEE Trans on CAD of Int. Circuits \& Systems, vol.27, no.2, pp.339-351, Feb. 2008

[8] Stratigopoulos, H.-G.; Mir, S., "Adaptive alternate analog test," IEEE Design \& Test of Computers, pp.71-79, July/August 2012

[9] Ayari, H.; et al.; "On the use of redundancy to reduce prediction error in alternate analog/RF test", IEEE Int'l Mixed-Signals, Sensors \& Systems Test Workshop (IMS3TW), pp.34-39, May 2012.

[10] Ayari, H.; et al.; "Making predictive analog/RF alternate test strategy independent of training set size", IEEE Int'l Test Conf. (ITC), paper 10.1, 2012 